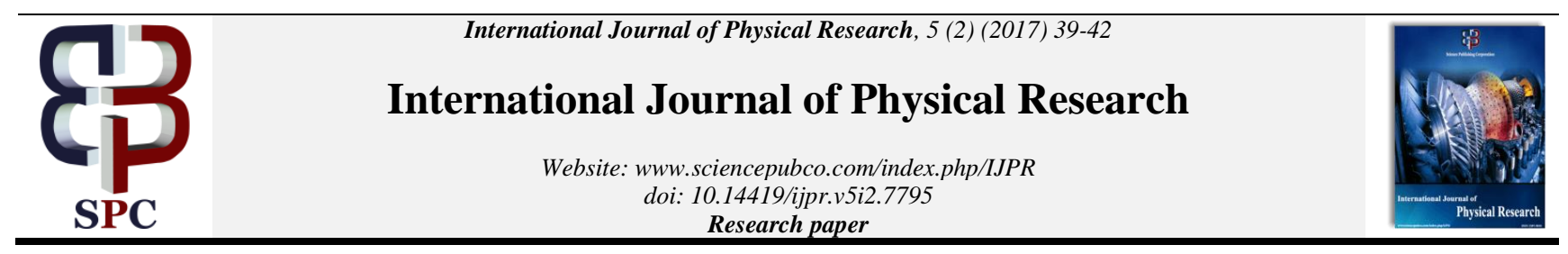

\title{
Bright and dark 1- soliton solutions to perturbed Schrodinger - hirota equation with power law nonlinearity via semi - inverse variation method and ansatz method
}

\author{
S. Subhaschandra Singh * \\ Physics Department, Imphal College, Imphal - 795001,Manipur, India \\ *Corresponding authorE-mail: subhasic@yahoo.co.in
}

\begin{abstract}
This paper studies perturbed Schrodinger Hirota equation with power law nonlinearity by obtaining its 1 - soliton solutions via He's semi - inverse variation method and the Ansatz method and the results reveal that these methods are very effective ones for obtaining exact solutions to various types of nonlinear evolution equations appearing in the studies of science and engineering.
\end{abstract}

Keywords: Fiber Optics; Optical Soliton; Group Velocity Dispersion (GVD); Third Order Dispersion (TOD).

\section{Introduction}

During the past a few decades, studies of optical solitons have become the most important branch of study in the field of Fiber Optics. Solitons are the result of a delicate balance between dispersion and nonlinearity. In particular, optical solitons are pulses which act as information carriers through optical fibers for Trans continental and Trans - oceanic distances. For such signal transmissions, the basic governing equation is the so-called Nonlinear Schrodinger Equation (NLSE) [1], [2]. When dispersive effect like the third order dispersion (TOD) is taken into account, in addition to the group velocity dispersion (GVD), the nonlinear Schrodinger equation can be transformed into a nonlinear evolution equation known as the Schrodinger - Hirota equation (SHE) [3 - 7] through the application of Lie symmetry. When perturbation terms are taken into consideration, a perturbed Schrodinger - Hirota equation (perturbed SHE) with a power law nonlinearity can be written in the form

$$
\begin{aligned}
& i q_{t}+a q_{x x}+b|q|^{2 n} q+i c q_{x x x}+i c \lambda|q|^{2 n} q_{x} \\
& =i \alpha q_{x}+i \beta\left(|q|^{2 n} q\right)_{x}+i \gamma\left(|q|^{2 n}\right)_{x} q
\end{aligned}
$$

where the independent variables $\mathrm{x}$ and $\mathrm{t}$ are spatial and temporal variables respectively, $\mathrm{x}$ being the displacement in the direction of wave propagation and the retarded time in the GVD frame, the number $\mathrm{n}$ dictates the power law nonlinearity, the dependent variable $\mathrm{q}(\mathrm{x}, \mathrm{t})$ is a complex valued function representing a wave profile and the subscripts denote partial derivatives with respect to them. On the left hand side, the first term is the time evolution term, the second is the GVD term, the third is a term with power law nonlinearity, the fourth is the third order dispersion (TOD) term and the fifth is a nonlinear dispersion (NLD) term. On the right hand side, the first term is the inter-modal dispersion term, the second one is the self-steepening (SS) term and the last one is another version NLD term. Moreover, $a, b, c, \lambda, \alpha, \beta, \gamma$ are constants and $i=\sqrt{-1}$ is imaginary number. For power law nonlinearity, it is necessary to have the restriction $0<\mathrm{n}<2$ in order to avoid soliton collapse and in particular $n \neq 2$ in order to avoid self - focusing singularity in nonlinear optics.

\section{Reduction to nonlinear ordinary differential equation}

To reduce Eq. (1) to a nonlinear ordinary differential equation, let us introduce the transformations

$q(x, t)=u(\xi) e^{i(-k x+\omega t+\phi)}, \xi=x-v t$

where $\mathrm{k}$ is the constant frequency of the soliton, $\omega$ is the constant soliton wave number $\varphi$ is a phase constant and $\mathrm{v}$ is the constant speed of the soliton.

Substituting $\mathrm{q}(\mathrm{x}, \mathrm{t})$ from $\mathrm{Eq} .(2)$ and also its relevant partial derivatives into Eq.(1) and then splitting the resulting equation into real and imaginary parts, we write

Real Part:

$$
\begin{aligned}
& (a+3 c k) \frac{d^{2} u}{d \xi^{2}}-\left(\omega+\alpha k+a k^{2}+c k^{3}\right) u \\
& +(b+c \lambda k-\beta k) u^{2 n+1}=0
\end{aligned}
$$

and Imaginary Part:

$$
\begin{aligned}
& c \frac{d^{3} u}{d \xi^{3}}-\left(v+\alpha+2 a k+3 c^{2}\right) \frac{d u}{d \xi} \\
& +\{c \lambda-(2 n+1) \beta-2 n \gamma\} u^{2 n} \frac{d u}{d \xi}=0
\end{aligned}
$$


Integrating both sides of Eq.(4) once with respect to $\xi$ and choosing the integration constant as zero, we obtain

$c \frac{d^{2} u}{d \xi^{2}}-\left(v+\alpha+2 a k+3 c k^{2}\right) u$

$+\{c \lambda-(2 \mathrm{n}+1) \beta-2 \mathrm{n} \gamma\} \frac{\mathrm{u}^{2 \mathrm{n}+1}}{(2 \mathrm{n}+1)}=0$.

Since Eqs. (3) and (5) are simultaneously satisfied by the same function $u(\xi)$, we must have the constraint conditions

$\frac{\mathrm{a}+3 \mathrm{ck}}{\mathrm{c}}=\frac{\omega+\alpha \mathrm{k}+\mathrm{ak}^{2}+\mathrm{ck}^{3}}{\mathrm{v}+\alpha+2 \mathrm{ak}+3 \mathrm{ck}^{2}}$

$=\frac{(2 n+1)(b+c \lambda k-\beta k)}{c \lambda-(2 n+1) \beta-2 n \gamma}$

Now, instead of solving both of the equations (3) and (5), we can solve any one of these two. Here, let us solve Eq. (5).

\section{Outlines of semi-inverse variation method (SVM)}

In this section, the outlines of He's SVM [8 - 13] is presented as in the following. If the nonlinear partial differential equation to be solved is in the form

$P\left(u, u_{t}, u_{x}, u_{t t}, u_{t x}, u_{x x}, \ldots\right)=0$

where $t$ and $x$ are independent variables and the subscripts denote partial derivatives of the dependent variable $u$ with respect to them, then we are to perform the following operational steps.

Step 1: The given nonlinear partial differential equation (NLPDE) is first to be reduced to a nonlinear ordinary differential equation (NLODE) by introducing a transformation $\mathrm{u}(\mathrm{x}, \mathrm{t})=\mathrm{U}(\xi)$ and $\xi=$ $\mathrm{x}-\mathrm{vt}$ where $\mathrm{v}$ is a constant, generally, the constant speed of wave propagation. Let us assume that the equation is now reduced to a NLODE as

$\mathrm{Q}\left(\mathrm{U}, \mathrm{U}^{\prime}, \mathrm{U}^{\prime \prime}, \ldots\right)=0$

where

$U^{\prime}=\frac{d U}{d \xi}, U^{\prime \prime}=\frac{d^{2} U}{d \xi^{2}}, \ldots e t c$

Step 2: The reduced NLODE is to be integrated once or more so long as every term in that equation contains derivative(s) and for the sake of simplicity, we are to choose the integration constant(s) as zero.

Step 3: Next, we are to construct a trial function

$J(U)=\int_{a}^{b} L d \xi$

where $a$ and $b$ are the lower and the upper limits related to the problem and $\mathrm{L}$ is a function of $\mathrm{U}$ and its derivatives.

Step 4: In accordance with Ritz method, we assume a solitary wave solution $\mathrm{U}$ in any one of the forms

$U(\xi)=A \operatorname{sech}^{p}(B \xi), U(\xi)=A \operatorname{cosech}^{p}(B \xi)$,

$U(\xi)=A \tanh ^{p}(B \xi), U(\xi)=A \operatorname{coth}^{p}(B \xi)$,

etc.

Here, the parameter $\mathrm{p}$ is to be determined through a balancing of degrees between the term with the highest derivative and the term with highest nonlinearity. The remaining parameters A and B are to be determined by substituting the assumed solitary solution to
Eq. (9) and making $J$ stationary with respect to $A$ and $B$, that is making

$\frac{\partial J}{\partial A}=0$

and

$\frac{\partial J}{\partial B}=0$

Solving Eqs. (10) and (11), we will obtain the values of the parameters $\mathrm{A}$ and $\mathrm{B}$. Then, substituting the values of $\mathrm{p}, \mathrm{A}$ and $\mathrm{B}$ into the assumed solitary wave solution, we can obtain a solution of the Reduced NLODE and hence of the given NLPDE.

\section{Application of semi-inverse variation meth- od}

Multiplying both sides of Eq. (5) by $\frac{d U}{d \xi}$ and integrating the result once with respect to $\xi$, we define

$S=-c\left(\frac{d U}{d \xi}\right)^{2}-\left(v+\alpha+2 a k+3 c k^{2}\right) U^{2}$

$+\{c \lambda-(2 n+1) \beta-2 n \gamma\} \frac{U^{2 n+2}}{(n+1)(2 n+1)}$.

We define a stationary integral by

$J=\int_{-\infty}^{+\infty} S d \xi$

$=\int_{-\infty}^{+\infty}\left[\begin{array}{c}-c\left(\frac{d U}{d \xi}\right)^{2} \\ -\left(v+\alpha+2 a k+3 c k^{2}\right) U^{2} \\ +\{c \lambda-(2 n+1) \beta-2 n \gamma\} \frac{U^{2 n+2}}{(n+1)(2 n+1)}\end{array}\right] d \xi$.

From Equation (5), balancing of degrees between the term with highest order derivative and the term with highest nonlinearity results in

$p+2=p(2 n+1)$

giving

$p=\frac{1}{n}$

For power law nonlinearity, let us assume the 1-soliton solution as

$U(\xi)=A \operatorname{sech}^{\frac{1}{n}}(B \xi)$

where A is soliton amplitude and B is the soliton inverse width.

Substituting Eq. (15) into Eq. (13), we obtain

$J=\frac{\sqrt{\pi} \Gamma\left(\frac{1}{n}\right)}{\Gamma\left(\frac{1}{n}+\frac{1}{2}\right)}\left[\begin{array}{c}-\frac{c A^{2} B}{n(n+2)} \\ -\left(v+\alpha+2 a k+3 c k^{2}\right) \frac{A^{2}}{B} \\ +\frac{2\{c \lambda-(2 n+1) \beta-2 n \gamma\}}{(n+1)(n+2)(2 n+1)} \frac{A^{2 n+2}}{B}\end{array}\right]$.

Substituting Eq. (16) into Eq. (10), we obtain

$-\frac{c B^{2}}{n(n+2)}-\left(v+\alpha+2 a k+3 c k^{2}\right)$

$+\frac{2\{c \lambda-(2 n+1) \beta-2 n \gamma\}}{(n+2)(2 n+1)} A^{2 n}=0$.

Substituting Eq. (16) into Eq. (11), we obtain 
$-\frac{c B^{2}}{n(n+2)}+\left(v+\alpha+2 a k+3 c k^{2}\right)$

$-\frac{2\{c \lambda-(2 n+1) \beta-2 n \gamma\}}{(n+1)(n+2)(2 n+1)} A^{2 n}=0$.

Solving the two simultaneous equations (17) and (18), we obtain

$A= \pm\left[\frac{(n+1)(2 n+1)\left(v+\alpha+2 a k+3 c k^{2}\right)}{c \lambda-(2 n+1) \beta-2 n \gamma}\right]^{\frac{1}{2 n}}$

and

$B= \pm n\left[\frac{v+\alpha+2 a k+3 c k^{2}}{c}\right]^{\frac{1}{2}}$

Substituting Eqs. (19) And (20) with positive roots into Eq. (15), we obtain

$U(\xi)=\left[\frac{(n+1)(2 n+1)\left(v+\alpha+2 a k+3 c k^{2}\right)}{c \lambda-(2 n+1) \beta-2 n \gamma}\right]^{\frac{1}{2 n}}$

$\times \operatorname{sech}^{\frac{1}{n}}\left\{n\left[\frac{v+\alpha+2 a k+3 c k^{2}}{c}\right]^{\frac{1}{2}} \xi\right\}$

Substituting Eq. (21) into Eq. (2), we write the bright 1-soliton solution of Eq. (1) as

$q(x, t)$

$=\left[\frac{(n+1)(2 n+1)\left(v+\alpha+2 a k+3 c k^{2}\right)}{c \lambda-(2 n+1) \beta-2 n \gamma}\right]^{\frac{1}{2 n}}$

$\times \operatorname{sech}^{\frac{1}{n}}\left\{n\left[\frac{v+\alpha+2 a k+3 c k^{2}}{c}\right]^{\frac{1}{2}}(x-v t)\right\}$

$\times e^{i(-k x+\omega t+\phi)}$.

with the constraints given in (6).

\section{Ansatz method}

In this section, Bright (or Non - topological) and Dark (or Topological) 1 - soliton solutions of the perturbed Schrodinger - Hirota equation (SHE) are obtained via the solitary wave ansatz method [14-16].

\subsection{Bright 1- soliton Solution}

For bright 1 - soliton solution, we assume a solution of Eq. (5) in the form

$$
U(\xi)=A \operatorname{sech}^{p}(B \xi)
$$

where A, p and B are constants to be determined. Here, A and B are respectively called the soliton amplitude and the soliton inverse width.

Substituting $U(\xi)$ from Eq. (23) and its relevant derivatives into Eq. (5), we obtain

$$
\begin{aligned}
& c\left[p^{2} A B^{2} \operatorname{sech}^{p}(B \xi)-p(p+1) A B^{2} \operatorname{sech}^{p+2}(B \xi)\right] \\
& -\left(v+\alpha+2 a k+3 c k^{2}\right) A \operatorname{sech}^{p}(B \xi) \\
& +\{c \lambda-(2 n+1) \beta-2 n \gamma\} \frac{A^{2 n+1}}{2 n+1} \operatorname{sech}^{p(2 n+1)}(B \xi) \\
& =0 .
\end{aligned}
$$

From Eq. (24), equating the exponents $p+2$ and $p(2 n+1)$, we obtain

$p=\frac{1}{n}$

Now, from Eq. (24), equating the coefficient of $\operatorname{sech}^{2+\frac{1}{n}}(B \xi)$ to zero and solving for $\mathrm{B}$, we obtain

$B=n A^{n}\left[\frac{c \lambda-(2 n+1) \beta-2 n \gamma}{c(n+1)(2 n+1)}\right]^{\frac{1}{2}}$.

Again, from Eq. (24), equating the coefficient of $\operatorname{sech}^{\frac{1}{n}}(B \xi)$ to zero and solving for $\mathrm{v}$, we obtain

$v=\frac{c B^{2}}{n^{2}}-\left(\alpha+2 a k+3 c k^{2}\right)$.

Here, from Eq. (26), we have another constraint condition viz.

$c\{c \lambda-(2 n+1) \beta-2 n \gamma\}>0$.

Substituting p and B in Eq. (23), we obtain the solution of Eq. (5) as

$U(\xi)=A \operatorname{sech}^{\frac{1}{n}}\left\{n A^{n}\left[\frac{c \lambda-(2 n+1) \beta-2 n \gamma}{c(n+1)(2 n+1)}\right]^{\frac{1}{2}} \xi\right\}$

Substituting Eq. (29) into Eq. (2), we obtain the bright 1 - soliton solution of Eq. (1) as

$q(x, t)$

$=A \operatorname{sech}^{\frac{1}{n}}\left\{n A^{n}\left[\frac{c \lambda-(2 n+1) \beta-2 n \gamma}{c(n+1)(2 n+1)}\right]^{\frac{1}{2}}(x-v t)\right\}$

$\times e^{i(-k x+\omega t+\phi)}$

with the constraint conditions shown in (6) and (28).

Here, $\mathrm{A}$ is a running parameter.

\subsection{Dark 1- soliton solution}

For dark 1 - soliton solution, we assume a solution of Eq. (5) in the form

$U(\xi)=A \tanh ^{p}(B \xi)$

where A, p and B are constants to be determined. Here, A and B are respectively called the soliton amplitude and the soliton inverse width.

Substituting $U(\xi)$ from Eq. (31) and its relevant derivatives into Eq.(5), we obtain

$c\left[A B^{2} p(p-1)\right] \tanh ^{p-2}(B \xi)$

$-2 \mathrm{p}^{2} A B^{2} \tanh ^{p}(B \xi)+A B^{2} p(p+1) \tanh ^{p+2}(B \xi)$

$-\left(v+\alpha+2 a k+3 c k^{2}\right) A \tanh ^{p}(B \xi)$

$+\{c \lambda-(2 n+1) \beta-2 n \gamma\} \frac{A^{2 n+1}}{2 n+1} \tanh ^{p(2 n+1)}(B \xi)$

$=0$.

From Eq. (32), equating the exponents $p+2$ and $p(2 n+1)$, we obtain

$p=\frac{1}{n}$. 
Again, from Eq. (32), equating the coefficient of $\tanh ^{p-2}(B \xi)$ to zero, we obtain

$p=\frac{1}{n}=1$ giving $\mathrm{n}=1$.

Moreover, from Eq. (32), equating the coefficient of $A \tanh ^{3}(B \xi)$ to zero, we obtain

$B=A\left[\frac{3 \beta+2 \gamma-c \lambda}{6 c}\right]^{\frac{1}{2}}$.

Substituting the values of p and B into Eq. (31), we obtain a solution of Eq.(5) as

$U(\xi)=A \tanh \left\{A\left[\frac{3 \beta+2 \gamma-c \lambda}{6 c}\right]^{\frac{1}{2}} \xi\right\}$

Here, $\mathrm{A}$ is a running parameter as before.

Substituting Eq. (36) into Eq. (2), we obtain the dark 1 - soliton solution of Eq. (1) as

$q(x, t)=A \tanh \left\{A\left[\frac{3 \beta+2 \gamma-c \lambda}{6 c}\right]^{\frac{1}{2}}(x-v t)\right\}$

$\times e^{i(-k x+\omega t+\phi)}$.

\section{Discussion}

It is observed that the semi - inverse variation method (SVM) can yield only the bright soliton solution whereas the ansatz method can yield both the bright and the dark soliton solutions. Moreover, the ansatz method reveals the fact that the dark soliton solution for the perturbed Schrodinger - Hirota equation exists only when the power law nonlinearity reduces to the Kerr law nonlinearity. We know that a medium that exhibits Kerr law nonlinearity is one in which the intensity of light passing through it depends on its refractive index.

\section{Concluion}

In this paper, bright and dark soliton solutions of perturbed Schrodinger - Hirota equation have been obtained and the results show that the methods used here are effective tools for obtaining exact solutions of various types of nonlinear evolution equations (NLEEs) encountered in the study of many areas of nonlinear science and engineering.

\section{References}

[1] A. Chabachoub, B.Kibler, C. Finot, G. Milot, M. Onoroto, J.M Dudley and A.V. Babanin, The nonlinear Schrodinger equation and the propagation of weakly nonlinear waves in optical fibers and on the water surface, Ann. Phys. 361, 490 - 500 (2015). https://doi.org/10.1016/j.aop.2015.07.003.

[2] D. Chiron, Travelling waves for the nonlinear Schrodinger equation with general nonlinearity in dimension one, Nonlinearity 25,813 850 (2012). https://doi.org/10.1088/0951-7715/25/3/813.

[3] A. Biswas, M. Mirzazadeh and M.Eslami, Dispersive dark optical soliton with Schrodinger - Hirota equation by G//G - expansion approach in power law medium, Optik 125, 4215 - 4218 (2014). https://doi.org/10.1016/j.ijleo.2014.03.039

[4] A. Biswas, A. J. M. Jawad, W. N. Manrakhan, A. K. Sarma and K R. Khan, Optical solitons and complexitons of the Schrodinger Hirota equation, OPT LASER TECHNOL, 44(7) 2265 - 2269 (2012). https://doi.org/10.1016/j.optlastec.2012.02.028.

[5] A. J. M. Jawad, The sine - cosine function method for the exact solutions of the nonlinear partial differential equations, IJRRAS 13(1), 186 - 191 (2012)
[6] A. J. M. Jawad, S. Kumar and A. Biswas, Soliton solutions of a few nonlinear wave equations in engineering sciences, SCI IRAN D 21(3), 861 - 869 (2014).

[7] M. Akbari, the Modified Simple Equation Method for Finding the Exact Solutions of Nonlinear PDEs in Mathematical Physics, Quant.Phys. Lett. 3(3), 33 -36 (2014).

[8] A. Biswas, Soliton Solutions of the Perturbed Resonant Nonlinear Schrodinger Equation with Full Nonlinearity by Semi-inverse Variational Principle, Quant. Phys.Lett. 1(2), 79 - 83 (2013).

[9] S. El - Ganaini, Applications of He's Variational principle and the First Integral Method to the Gardner Equation, Appl. Math. Sci., 6(86), 4249 - 4260 (2012).

[10] A. Jabbari, H. Kheiri and A. Bekir, Exact solutions of the coupled Higgs equation and the Maccari system using He's semi - inverse method and ( expansion method, Comput. Math. Appl., 62, 2177 2186 (2011). https://doi.org/10.1016/j.camwa.2011.07.003.

[11] H. Kheir, A. Jabbari, A. Yildrim and A. K. Alomari, He's semiinverse method for soliton solutions of Boussinesq system, World Journal of Modelling and Simulation, 9(1), 30 13, (2013).

[12] M. Mirzazadeh, Topological and non-topological soliton solutions of Hamiltonian amplitude equation by He's semi-inverse method and ansatz approach, Journal of Egyptian Mathematical Society, 23 292 - 296 (2015). https://doi.org/10.1016/i.joems.2014.06.005.

[13] M. Najafi, S. Arbabi and M. Najafi, He's semi-inverse method for Camassa-Holm equation and simplified modified Camassa-Holm equation, International Journal of Physical Research 1(1), 1 - 6 (2013). https://doi.org/10.14419/ijpr.v1i1.679.

[14] O. Guner, Bright and dark soliton solution of the nonlinear partial differential equations system, New Trends in Mathematical Sciences, 3(4), $154-163$ (2015).

[15] M. Mirzazadeh, Topological and non-topological soliton solutions of Hamiltonian amplitude equation by He's semi-inverse method and ansatz approach, Journal of Egyptian Mathematical Society, 23, 292 - 296 (2015). https://doi.org/10.1016/j.joems.2014.06.005.

[16] H. Triki, A. Yildrim, T. Hayat, O. M. Aldossar and A. Biswas, Topological and non-topological soliton solutions of the Bertherton equation, Proceedings of the Romanian Academy Series A, vol. 13, No. 2, 103 - 108 (2012) 